Cahiers $d u$ MONDE RUSSE

\section{Cahiers du monde russe}

Russie - Empire russe - Union soviétique et États indépendants

48/2-3 | 2007

Les résonances de 1905

\title{
Des censeurs aux inspecteurs de la presse
}

crise et métamorphoses des organes de censure après 1905

\section{Benjamin Guichard}

\section{(2) OpenEdition}

1 Journals

Édition électronique

URL : https://journals.openedition.org/monderusse/9007

DOI : 10.4000/monderusse. 9007

ISSN : $1777-5388$

Éditeur

Éditions de l'EHESS

\section{Édition imprimée}

Date de publication : 15 avril 2007

Pagination : 331-346

ISBN : 978-2-7132-2147-7

ISSN : $1252-6576$

\section{Référence électronique}

Benjamin Guichard, "Des censeurs aux inspecteurs de la presse », Cahiers du monde russe [En ligne], 48/2-3 | 2007, mis en ligne le 01 janvier 2007, consulté le 04 septembre 2022. URL : http:// journals.openedition.org/monderusse/9007; DOI : https://doi.org/10.4000/monderusse.9007 
chercher : repérer : avancer

Cet article est disponible en ligne à l'adresse :

http://www.cairn.info/article.php?ID REVUE=CMR\&ID NUMPUBLIE=CMR 482\&ID ARTICLE=CMR 4820331

Des censeurs aux inspecteurs de la presse. Crise et métamorphoses des organes de censure après 1905

\author{
par Benjamin GUICHARD
}

\title{
Editions de l'EHESS | Cahiers du monde russe
}

\section{7/2-3 - Vol 48}

ISSN 1252-6576 | ISBN 9782713221477 | pages 331 à 346

Pour citer cet article :

- GUICHARD B., Des censeurs aux inspecteurs de la presse. Crise et métamorphoses des organes de censure après 1905, Cahiers du monde russe 2007/2-3, Vol 48, p. 331-346.

Distribution électronique Cairn pour les Editions de l'EHESS.

(C) Editions de l'EHESS. Tous droits réservés pour tous pays.

La reproduction ou représentation de cet article, notamment par photocopie, n'est autorisée que dans les limites des conditions générales d'utilisation du site ou, le cas échéant, des conditions générales de la licence souscrite par votre établissement. Toute autre reproduction ou représentation, en tout ou partie, sous quelque forme et de quelque manière que ce soit, est interdite sauf accord préalable et écrit de l'éditeur, en dehors des cas prévus par la législation en vigueur en France. Il est précisé que son stockage dans une base de données est également interdit. 


\title{
DES CENSEURS AUX INSPECTEURS DE LA PRESSE
}

\author{
Crise et métamorphoses des organes \\ de censure après 1905
}

La révolution de 1905-1906 marque un tournant dans l'histoire du livre et de la presse russes avec l'abandon de la censure préventive au profit d'une censure répressive, postérieure à la publication et sanctionnée par le pouvoir judiciaire. Ce système, élaboré lors des réformes de 1865 et alors réservé aux capitales, n'est en effet mis en œuvre à une grande échelle qu'avec les règlements provisoires de novembre 1905 et du printemps 1906 qui étendent les procédures de contrôle a posteriori à l'ensemble des livres et des périodiques publiés dans les villes de l'Empire. Cette législation est directement issue des promesses formulées dans les manifestes du 12 décembre 1904 et du 17 octobre $1905^{1}$, mais elle maintient différents régimes spéciaux hérités du siècle précédent. Ce caractère hybride, entre concessions libérales et traditions réactionnaires, marque une volonté de protéger les prérogatives de l'État tout en reconnaissant la nécessité de donner des gages d'ouverture.

L'historiographie a ainsi souvent donné une image très contrastée du régime de la presse après 1905. La recherche soviétique, en s'appuyant sur le sort des journaux socialistes, défendait l'idée d'une censure tsariste violemment répressive et marginalement modifiée après $1905^{2}$. Les chercheurs russes s'intéressent encore

1. Marc Szeftel, «Le Manifeste du 17 octobre et son rôle dans l'évolution constitutionnelle de l'Empire », in François-Xavier Coquin, Céline Gervais-Francelle, éd., 1905: la première révolution russe, P. : Publications de la Sorbonne - Institut d'Études slaves, 1986, p. 13-30.

2. A.F. Berežnoj, Carskaja cenzura i bor’ba bolševikov za svobodu pečati, 1895-1914 [La censure tsariste et la lutte des bolcheviques pour la liberté de la presse, 1895-1914], L. : izdatel'stvo Leningradskogo universiteta, 1967, p. 180-218. 
peu aux particularités de l'institution après le manifeste du 17 octobre $^{3}$. Les historiens occidentaux ont défendu à l'inverse la thèse d'une modération de la censure après 1905, mais moins par une volonté délibérée du régime qu'en raison des dysfonctionnements des organes de contrôle. Benjamin Rigberg a dressé le portrait d'une censure peu efficace tout au long du XIX siècle se trouvant paralysée par l'abolition de la censure préventive et la stagnation de ses moyens humains ${ }^{4}$. Cette vision explique que, dans l'historiographie occidentale, les études les plus substantielles sur le contrôle de la presse s'arrêtent à la réforme de 1906, l'institution ne semblant plus jouer ensuite qu'un rôle anecdotique ${ }^{5}$ Caspar Ferenczi a affiné l'analyse et montré que la libéralisation correspondait à un choix de l'État soucieux d'utiliser la presse comme médiation entre le pouvoir et le peuple pour remplacer les institutions traditionnelles de l'Église ou du charisme impérial ${ }^{6}$. Il a également cherché à comprendre le décalage souligné par Jacob Walkin ${ }^{7}$ entre pratiques et perceptions de la censure, en montrant que la répression agissait de façon sélective selon les priorités politiques du régime. Son analyse permet ainsi de réconcilier partiellement la vision très sombre des historiens soviétiques et l'appréciation des chercheurs anglo-saxons. Dans un essai de synthèse bien informé, Jonathan Daly invite cependant à relativiser le poids de la réglementation : pour lui, le recul de la presse radicale après 1907 et de la presse libérale après 1910 ne s'expliquent pas tant par la pression de la censure que par la concurrence d'une presse populaire dépolitisée ${ }^{8}$. L'ampleur du changement dans les pratiques et les normes de la censure après 1905 reste ainsi largement discutée.

Le débat historiographique s'est donc organisé autour de la question de la sévérité et de l'efficacité de la censure en fonction du nombre de poursuites judiciaires et de fermetures de journaux. Cette description externe des effets et des formes de la censure éclaire trop partiellement la transformation des organes de contrôle. Nous proposons ici de nous pencher sur les conceptions que les censeurs avaient de leur rôle après 1905 et leur perception de la réforme. Celle-ci impliquait la modification du nom de l'institution et de ses acteurs.

3. Natalja G. Patruševa, «Izučenie istorii cenzury vtoroj poloviny XIX-načala XX v. v 19601990-e gg. (Bibliografičeskij obzor) » [L'historiographie de la censure de la deuxième moitié du XIX ${ }^{\mathrm{e}} \mathrm{s}$. et du début du XXe s. dans les années 1960-1990. (Panorama bibliographique)], Novoe literaturnoe obozrenie, 1998, 30, p. 427-428.

4. Benjamin Rigberg, « The Efficacy of Tsarist Censorship Operations, 1884-1917 », Jahrbücher für Geschichte Osteuropas, 14, 1966, p. 327-346.

5. Charles Ruud, Fighting Words : Imperial Censorship and the Russian Press 1804-1906, Toronto : University of Toronto Press, 1982, 327 p. ; Daniel Balmuth, Censorship in Russia 1865-1905, Washington D.C. : University Press of America, 1979, 249 p. ; Marianna Tax Choldin, A Fence around the Empire : Censorship of Foreign Ideas under the Tsars, Durham : Duke University Press, 1985, 281 p.

6. Caspar Ferenczi, « Freedom of the Press under the Old Regime, 1905-1914 », in Olga Crisp, Linda Edmondson, éd., Civil Rights in Imperial Russia, Oxford : Clarendon, 1989, p. 191-214.

7. Jacob Walkin, «Government Control over the Press in Russia 1905-1914 », Russian Review, 13(3), 1954, p. 203-209.

8. Jonathan Daly, «Pressa i gosudarstvo v Rossi (1906-1917 gg.) » [La presse et l'État en Russie (1906-1917)], Voprosy istorii, 10, 2001, p. 25-45. 
Le terme de « censure » était utilisé depuis 1796 pour désigner l'administration chargée du contrôle de la presse ${ }^{9}$, mais les auteurs des réformes de 1865 avaient choisi d'abandonner ce mot pour désigner les instances centrales, baptisées Administration générale des affaires de presse (Glavnoe upravlenie po delam pečati). Les termes de censure et de censeur restaient cependant utilisés pour désigner les échelons locaux (cenzurnye komitety) et le personnel. L'oukase du 26 avril 1906 fait disparaitre les dernières traces de ce mot au profit des expressions « comités aux affaires de presse » et « inspecteurs des affaires de presse ${ }^{10}$. L'initiative a été raillée par les contemporains. Le militant KD Gorbunov dénonce une innovation «purement sémantique » qui masque le maintien d'un pouvoir discrétionnaire de l'administration : «Si la censure préventive est abolie, à quoi bon conserver les censeurs et les comités de censure, même sous un nouveau nom, et à quoi bon contraindre les éditeurs et les imprimeurs à leur remettre des exemplaires de leurs publications ? ${ }^{11}$. Cette euphémisation ne doit cependant pas être considérée comme vide de sens. La décision révèle la prise de conscience au moins partielle du caractère illégitime de la censure qu'il faut donc masquer sous un nouveau nom. Pour le Conseil d'État, la décision est justifiée par l'évolution du fonctionnement du contrôle de la presse qui « se limitera désormais à instruire des poursuites judiciaires »12. La transformation des censeurs en « inspecteurs de la presse » peut ainsi être interprétée comme le symptôme d'une crise d'identité professionnelle provoquée par les événements de 1905-1906. Celle-ci se développe dans les cadres de la nouvelle réglementation et incite les censeurs à faire évoluer les méthodes et les missions de leur institution.

\section{Une crise d'identité professionnelle révélée par les événements de 1905}

Les organes de censure étaient déjà en crise lorsque les événements politiques de 1905 éclatèrent. L'abandon du programme de réforme de 1865 et la multiplication des procédures autoritaires en matière de la presse introduites au cours des années 1880 suscitaient l'opprobre des hommes de presse de tous courants. L'article 140 du statut de la censure ${ }^{13}$, qui donnait le pouvoir au ministère de l'Intérieur d'interdire par circu-

9. L.V. Volkov, «Cenzura» [Censure], Gosudarstvennost` Rossii (konec XV v.-fevral` 1917 g.) [L’État en Russie (fin du XVe siècle-février 1917)], t. 4, M. : Nauka, 1996, p. 387-388.

10. Articles 2 et 3 de l'oukase du 26 avril 1906, «O vremennyh pravilah dlja nepovremennoj pečati » [Réglementation provisoire de la presse non-périodique], Sobranie uzakonenij i rasporjaženij pravitel'stva [Recueil de lois et des ordonnances du gouvernement], 3 mai 1906, article 637.

11. A.V. Gorbunov, «Dejstvujuščie zakony o pečati » [Les lois en vigueur sur la presse], in Pečat' pri obnovlennom stroe [La liberté de la presse à l'heure du nouveau régime], $\mathrm{SPb}$. : Obščestvennaja pol'za, 1912,p. 71 et 73.

12. OR RNB (Otdel rukopisej Rossijskoj nacional'noj biblioteki - Département des manuscrits de la Bibliothèque nationale de Russie), f. 781, fonds I. I. Tolstoj, d. 296, 1. 28, compte rendu des débats du Conseil d'État du 4 avril 1906.

13. «Ustav o cenzure i pečati (izd. 1890 g.) » [Statut de la censure et de la presse (éd. de 1890)], Svod zakonov Rossijskoj Imperii [Corps de lois de 1'Empire russe], SPb : Gosudarstvennaja tipografija, s.d., t. XIV. 
laires la mention dans les journaux d'événements ponctuels et de débattre de certains problèmes politiques et sociaux, concentrait l'hostilité. Celle-ci, par exemple, s'était exprimée à travers une pétition d'hommes de lettres présentés à Nicolas II au début de son règne ${ }^{14}$. Mais il faut attendre la guerre russo-japonaise, et la multiplication de remises en cause directes du pouvoir dans les journaux, pour que le gouvernement mette la réforme de la presse à l'ordre du jour. À la fin janvier 1905, le ministre de l'Intérieur A.G. Bulygin expose à Nicolas II l'impasse de la logique répressive et propose de lutter contre les publications révolutionnaires avec de nouvelles armes : « la littérature clandestine n'a pas les moyens de répondre à la libre critique, c'est donc par cette voie qu'il faut la paralyser $\gg^{15}$. La réforme de la censure est donc motivée moins par une volonté d'ouverture libérale que par le constat d'inefficacité du régime policier et disparate progressivement mis en place au cours des quarante années précédentes. Ce programme est formulé une première fois dans l'oukase du 12 décembre 1904 qui dénonce, entre autres, les restrictions abusives dont la presse est la victime sans énoncer le principe de liberté d'expression. Ce texte débouche sur la convocation d'une commission chargée de rédiger une nouvelle loi sur la presse, présidée par le directeur de la bibliothèque impériale publique, D.F. Kobeko. Le projet rassemble les représentants de différents ministères, du Saint-Synode, les dirigeants de grands groupes de presse comme A.S. Suvorin, et des juristes, tels A.F. Koni et K.K. Arsen'ev. Seul le représentant du ministère de l'Intérieur, N.V. Šahovskoj, est un ancien cadre de l'Administration des affaires de presse qu'il a dirigée jusqu'en avril 1902. Les censeurs ne sont pas directement partie prenante de ce processus de réforme. Certains membres de la commission ont eu une brève expérience du contrôle de la presse, cependant, comme V.M. Juzefovič au contrôle des publications étrangères à Kiev, et le représentant du Saint-Synode, l'évèque de Narva Antonij, qui fut membre pendant quatre ans du comité de censure ecclésiastique de Saint-Pétersbourg. Mais cette expérience ne semble avoir joué qu'un rôle incident dans leur nomination et ils n'en font pas état au cours des débats ${ }^{16}$. Le rôle des membres des organes de contrôle se limite donc à celui d'experts auditionnés par la commission. La radicalisation des événements politiques et le Manifeste du 17 octobre, proclamant pour la première fois la liberté de la presse, bouleversent le programme de la commission Kobeko ainsi que le travail de la censure.

Les censeurs sont immédiatement pris à partie par les éditeurs et les imprimeurs ne recevant, dans un premier temps, aucune instruction de la part de leur autorité de tutelle. Ainsi, à Odessa, le censeur Egorov est assailli de demandes et poursuivi

14. Petition of Russian Literary Men to Nicholas II for the Alleviation of the Censorship of the Press. Hodatajstvo russkih literatorov ob oblegčenii cenzury, Londres : Russian Free Press Fond, 1895, 32 p. ; Krasnyj arhiv, 20, 1927, p. 237-240.

15. Rapport présenté au tsar le 20 avril 1905 transcrit par I. Kovalev, Krasnyj arhiv, 105, 1941, p. 145 .

16. A.V. Lihomanov, «Osoboe soveščanie po sostavleniju novogo ustava o pečati 1905 g. : personal'nyj sostav » [La composition de la Commission spéciale pour l'établissement d'un nouveau statut de la presse de 1905], in B.N. Konašev, N.G. Patruševa, éd., Cenzura v Rossii : istorija i sovremennost' [La censure en Russie : histoire et actualité], t. 2, SPb : Rossijskaja nacional'naja biblioteka, 2005, p. 55, p. 63 . 
jusque dans la rue par des journalistes et des libraires qui s'étonnent, raconte-t-il, que « la censure existe encore et ne soit pas enterrée» ${ }^{17}$. Les autorités tentent cependant d'adapter le fonctionnement de la censure aux attentes de l'opinion dans une circulaire datée du 19 octobre $^{18}$. Même si le texte affirme que le statut de la presse continue à s'appliquer jusqu'à la mise en forme d'une nouvelle législation, il oriente les censeurs vers des accommodements. Le texte commence ainsi par offrir un argumentaire pro domo rappelant que dans tous les pays européens, la liberté de la presse s'accompagne d'une législation chargée de l'encadrer. La légitimité des censeurs ainsi défendue, il leur est cependant rappelé que leur comportement doit « radicalement changer » pour tenir compte des principes énoncés dans le manifeste impérial. Parallèlement, il est décidé de suspendre le contrôle discrétionnaire de la presse dont le ministère de l'Intérieur dispose en vertu de l'article $140 \mathrm{du}$ Statut de la censure. Les décisions des censeurs doivent désormais être strictement motivées en fonction des lois pénales du pays et les poursuites confiées au pouvoir judiciaire. Les réformes de 1865 sont donc rétablies à la lettre. Mais le texte exprime bien les doutes et les incertitudes de l'administration obligée d'agir dans l'urgence et dans un contexte hostile : « les censeurs doivent prendre en compte les nouvelles conditions qui s'imposent à la presse, faire usage de tout leur tact et éviter d'imposer des exigences qui ne sont pas assises sur le droit afin de ne pas susciter des réclamations justifiées ». Le texte du 19 octobre s'efforce de donner une assise juridique à la surveillance de la presse et marque ainsi une rupture essentielle qui prépare la généralisation de la censure répressive.

Le caractère soudain et brutal des grèves d'ouvriers typographes, les appels dès le 18 octobre au boycott de la censure par les éditeurs de Moscou, Saint-Pétersbourg et Odessa organisés en «Union pour la défense de la liberté de la presse », relayés par les syndicats de typographes et par les Soviets des deux capitales ${ }^{19}$ provoquent un traumatisme. À Odessa, au cours du mois d'octobre et du mois de novembre, les censeurs s'enferment à double tour dans leurs locaux que les manifestants menacent de mettre à $\operatorname{sac}^{20}$. Entre octobre 1905 et février 1906, plus de $85 \%$ des publications auraient été commercialisées sans respecter le dépôt légal selon le décompte de la commission chargée des questions d'enregistrement bibliographique auprès de l'Académie des sciences ${ }^{21}$. À Moscou, selon le pointage des autorités, vingt-deux éditeurs de livres, soit le nombre exact d'établissements

17. A.E. Egorov, «Stranicy iz prožitogo » [Pages vécues], in N.G. Patruševa, éd., Cenzura v Rossii v konce XIX-načale XX veka : Sbornik vospominanij [La censure fin XIX ${ }^{\mathrm{e}}$-début XX ${ }^{\mathrm{e}}$ siècle : recueil de souvenirs], SPb. : Dmitrij Bulanin, 2003,p. 155. Publication originale : Odessa, 1913.

18. Circulaire $n^{\circ} 11725$, citée d'après la version publiée dans Knižnyj vestnik, XXII, 44, 30 octobre 1905, col. 1251-1253. La fidélité du texte a été vérifiée avec l'exemplaire reçu par le Comité des affaires de presse de Finlande (Archives nationales de Finlande - Kansallisarkisto, KKK, Kenraalikuvernöörinkanslia, série Hd, Seynin asiakiriat, article 85, $1^{\text {re }}$ sous-chemise).

19. Marc Steinberg, Moral Communities : the Culture of Class Relations in the Russian Printing Industry, 1867-1907, Berkeley : University of California Press, 1992, p. 180-181.

20. Egorov, « Stranicy .... », p. 157.

21. Knižnyj vestnik, 23(26), 3 juillet 1906, col. 636. 
adhérents du syndicat de l'édition dans cette ville, ont boycotté la censure entre le 17 octobre et la mi-décembre $1905^{22}$.

Dans l'urgence, le gouvernement cherche d'abord à rétablir le contrôle des journaux. Une réglementation provisoire, en partie inspirée des travaux de la commission Kobeko, instaure formellement la censure répressive pour les publications périodiques de toutes les villes de l'Empire le 24 novembre ${ }^{23}$. Le texte est accueilli avec hostilité par les milieux de l'édition qui refusent de l'appliquer. La publication, le 2 décembre par les journaux des capitales, du manifeste du Soviet de Saint-Pétersbourg appelant au boycott fiscal pousse le gouvernement à réagir pour imposer la nouvelle réglementation par la force ${ }^{24}$. Malgré le grand nombre de poursuites, de confiscations et de fermetures de titres, l'autorité de la censure reste incertaine jusqu'au début du printemps 1906. À Saint-Pétersbourg, les censeurs sont ainsi contraints de se reposer sur la police pour traquer dans les librairies les livres publiés sans son aval25. Les édits des 24 novembre 1905 et 18 mars 1906 relatifs à la presse périodique, celui du 26 avril 1906 définissant les règles en vigueur pour les livres et les brochures mettent progressivement fin au boycott de la censure par les éditeurs et les imprimeurs. Mais ils ne règlent pas les difficultés des censeurs. En Finlande, la moitié des imprimeries du Grand-Duché continuent en 1911 à faire de la résistance passive en refusant de se soumettre au dépôt légal et d'adresser leurs ouvrages à la censure $^{26}$. Il s'agit cependant d'un cas extrême, car les pouvoirs locaux finlandais jouissent après 1905 d'une autonomie renouvelée ${ }^{27}$ et choisissent d'appuyer une interprétation libérale des réformes de la presse et de s'opposer, de façon frontale, aux restrictions imposées par le pouvoir central. Dans le reste de l'Empire, l'autorité de la censure est réaffirmée mais dans des formes nouvelles.

\section{Les conditions de travail des « inspecteurs de presse » sous le nouveau régime de la censure}

La nouvelle législation repose sur le double principe de la censure répressive et de la compétence exclusive de la justice pour punir les délits de presse. Les réactions

22. RGIA (Rossijskij Gosudarstvennyj Istoričeskij Arhiv - Archives historiques d'État de Russie), f. 776, Direction générale des affaires de presse (GUDP), op. 9, 1er Département, d. 97 , O knigah i brošjurah vypuščennyh bez predstavlenija na prosmotr cenzury, 1. 10-17, rapport de l'Inspecteur en chef des imprimeries de Moscou du 21 février 1906.

23. «O vremennyh pravilah o povremennoj pečati » [Réglementation provisoire de la presse périodique], Sobranie uzakonenij i rasporjaženij pravitel'stva, 26 novembre 1905, art. 1879.

24. Memuary grafa I.I. Tolstogo [Mémoires du comte I.I. Tolstoj], M. : Indrik, 2002, p. 217.

25. RGIA, f. 776, GUDP, op. 9, d. 97,1. 1, dépêche du préfet de Saint-Pétersbourg du 6 mars 1906.

26. Archives nationales de Finlande, KKK, Hd 85, rapport du Directeur général des affaires de presse pour la Finlande, V. Kannisten, du 12 mars 1912.

27. Antti Kujala, «Finland in 1905 : the political and social history of the revolution », in Jonathan Smele, Anthony Heywood, éd., The Russian Revolution of 1905 : Centenary Perspectives, Londres : Routledge, 2005, p. 89-93. 
contemporaines insistent sur les nombreuses entorses à ces principes fondamentaux dans la législation provisoire du printemps 1906 : maintien de régimes d'exception pour les ouvrages importés de l'étranger, les publications ayant trait à l'Empereur et les publications médicales ; maintien de la censure préventive pour les périodiques illustrés ${ }^{28}$ et instauration d'un délai de réserve pour les brochures de moins de 5 feuilles d'imprimerie (pečatnyj list) ${ }^{29}$, soit environ 80 pages au format in- $8^{\circ}$ ou 120 pages au format in- $12^{\circ 30}$, qui doivent être déposées auprès de la censure un à sept jours avant d'être distribuées dans le commerce. Ces restrictions ne doivent cependant pas faire oublier que le fonctionnement de la censure est profondément modifié par ces nouvelles procédures restées en vigueur jusqu'à la fin du régime.

La censure ne reçoit une copie des ouvrages périodiques et des livres qu'après l'impression de la totalité du tirage et la distribution peut en commencer dès l'envoi des exemplaires du dépôt légal. Ce principe de simultanéité, fortement contesté lors de l'examen du texte par le ministre de l'Intérieur P.N. Durnovo et une fraction du Conseil d'État ${ }^{31}$, est la clef de la nouvelle réglementation. S'il constate une infraction dans les ouvrages qui lui sont soumis, le censeur doit alors rédiger un rapport motivé au parquet pour lui demander d'engager des poursuites pénales. Seul le tribunal peut prononcer l'inculpation d'un éditeur et ordonner la destruction d'un imprimé. L'acte de censure, considéré désormais comme la répression d'un délit, est donc contrôlé par des magistrats. La décision s'inscrit dans un processus contradictoire, celui du procès, qui expose le censeur au désaveu. La Société des éditeurs et des libraires de Russie note ainsi avec satisfaction quelques semaines après que les premières affaires furent jugées que « malgré l'extrême lenteur de la procédure, les instances judicaires ont déjà cassé plusieurs poursuites contre des ouvrages 'subversifs' aboutissant ainsi à rendre des non-lieux en grande quantité au grand mécontentement des organes de contrôle de la presse $»^{32}$.

L'autorité du censeur est cependant préservée par son pouvoir de nuisance. Le commerce de la publication poursuivie est en effet interdit à titre conservatoire jusqu'au verdict judiciaire et les exemplaires détenus par l'imprimeur et l'éditeur confisqués. Cette saisie est confiée aux « inspecteurs des imprimeries », des fonctionnaires rattachés aux gouverneurs provinciaux. Le plus souvent, leur ordre de saisie est vain : l'imprimeur s'est empressé de diffuser au plus vite tout son tirage. Le censeur pétersbourgeois S.I. Umanec se souvient ainsi avec amertume : « À midi, les crieurs de journaux vendaient en toute liberté sur la perspective Nevski,

28. Article 3 de l'oukase du 18 mars 1906, « Ob izmenenii i dopolnenii vremennyh pravil o periodičeskoj pečati » [Modifications et compléments à la réglementation provisoire de la presse périodique], Sobranie uzakonenij i rasporjaženij pravitel'stva, 22 mars 1906, art. 428.

29. Article IV-2 de l'oukase du 26 avril 1906, « O vremennyh pravilah... ».

30. La feuille mesure par convention dans l'imprimerie russe 60 sur 90 centimètres.

31. Gilbert Doctorow, «Reforma carskoj cenzury » [La réforme de la censure tsariste], Kontinent, 36, 1983, p. 207-212.

32. «K massovym konfiskacijam knig » [Vers des confiscations massives de livres], Knižnyj vestnik,23(31), 7 août 1906, p. 706. 
aux principaux carrefours et à l'entrée des cafés les exemplaires que le comité venait de confisquer et le public se jetait sur eux $!{ }^{33}$.

Le censeur Egorov d'Odessa accueille cependant ces nouvelles procédures avec soulagement. Il voit dans l'abandon de la censure préventive des quotidiens la fin d'un « travail de Sisyphe » selon ses propres termes : lire chaque nuit la totalité des journaux du lendemain matin ${ }^{34}$. Il note avec plaisir que le censeur ne se verra plus reprocher le contenu des livres et des revues. Ils sont désormais publiés sans son accord et il est dégagé de toute responsabilité pénale à cet égard. Dans le système de la censure préventive, à l'inverse, tout éditeur poursuivi par les instances judiciaires ou policières pour un de ses ouvrages pouvait se retourner contre le censeur qui en avait autorisé la publication. Mais cet enthousiasme est de courte durée. Dès l'automne 1905, malgré le boycott par les éditeurs des deux capitales, les censeurs se plaignent de l'augmentation de leur charge de travail : les journaux du jour doivent être lus au plus vite après leur parution alors que le nombre de titres en circulation ne cesse d'augmenter ${ }^{35}$. Le Comité des affaires de presse de la capitale dénonce ainsi la fatigue nerveuse causée par les nouvelles procédures et estime que le nombre de publications contrôlées par chaque censeur a quadruplé entre 1905 et $1912^{36}$. L'efficacité des confiscations dépend en effet de la rapidité des censeurs qui doivent prendre de vitesse la distribution et la vente des imprimés. Chaque délit doit ensuite faire l'objet d'un rapport circonstancié transmis au parquet, ce qui ralentit encore la tâche des inspecteurs de presse. Le comité des affaires de presse de Saint-Pétersbourg dénonce ainsi à plusieurs reprises le manque de personnel pour expliquer le retard avec lequel il adresse aux magistrats les demandes de poursuites. Les censeurs se plaignent également de l'encombrement de leurs locaux par les sacs d'ouvrages confisqués en attente d'une décision de justice ${ }^{37}$.

Les confiscations prononcées par la censure sont donc en nombre limité et ont plus un pouvoir de nuisance pour les activités éditoriales qu'une réelle efficacité. Dès le 4 juin 1906, le président du Conseil I.L. Goremykin recommande à l'administration de ne pas se restreindre aux ressources de la nouvelle réglementation dans sa lutte avec la presse périodique d'opposition ${ }^{38}$. À compter de la dissolution de la Deuxième Douma le 3 juin 1907, le gouvernement recourt de façon systématique aux pouvoirs administratifs des gouverneurs ${ }^{39}$. Ces derniers peuvent utiliser

33. S. I. Umanec, «Iz prošlogo našej cenzury » [Épisode du passé de notre censure.], in Patruševa, éd., Cenzura v Rossii..., p. 201. Publication originale dans Naša starina, 1915, 10.

34. Egorov, « Stranicy ... », p. 158.

35. «Poslednie dni cenzury » [Les derniers jours de la censure], Knižnyj vestnik, 22(52), 31 décembre 1905, p. 1461-1462.

36. Rapport cité par Natalja G. Patruševa, « Istorija cenzurnyh učreždenij v Rossii vo vtoroj polovine XIX-načale XX v. » [Histoire des organes de censure en Russie dans la deuxième moitié du XIX ${ }^{\mathrm{e}}$ siècle et au début du XX ${ }^{\mathrm{e}}$ siècle], Knižnoe delo $v$ Rossii vo vtoroj polovine XIX -načale XX veka, 10,2000, p. 16.

37. Knižnyj vestnik, 23(35), 5 septembre 1906, p. 785 ; 24(39), 30 septembre 1907,p. 1207.

38. Rapport sur la presse présenté au tsar reproduit dans Krasnyj arhiv, 1922, 2, p. 280.

39. GARF (Gosudarstvennyj Arhiv Rossijskoj Federacii - Archives d'État de la Fédération de Russie), f. 102, Département de la police, op. 116, 4oe deloproizvodstvo (1907), d. 9233, 
le cadre des lois d'exception de 1881 pour prononcer par arrêtés des fermetures de journaux, d'imprimeries, des confiscations dans les librairies et bibliothèques de leur ressort. Les censeurs sont donc confrontés au transfert de fait d'une part de leurs tâches de contrôle du marché du livre et d'exécution des ordres de saisie aux autorités locales de police ${ }^{40}$. À Nijni-Novogorod, le gouverneur confisque ainsi systématiquement les publications poursuivies par la censure qui ont bénéficié d'un non-lieu devant la justice ${ }^{41}$. L'Administration générale des affaires de presse prodigue alors aux administrations provinciales un rôle de conseil pour la rédaction et la mise en œuvre de ces arrêtés ${ }^{42}$. Mais elle manifeste également, a contrario, un souci de respecter l'esprit de la nouvelle législation. La censure choisit ainsi d'abroger les restrictions aux acquisitions de livres dans les bibliothèques publiques qui étaient limitées à une liste d'ouvrages approuvés par les autorités. Elle décide en 1907 que tous les livres en vente qui n'ont pas été poursuivis par la censure peuvent être proposés aux lecteurs, précisant que cette mesure est une application « des nouvelles normes de droit, civiles et religieuses, proclamées dans le manifeste du 17 octobre $»^{43}$. Les relations entre l'Administration de la presse et la police oscillent ainsi entre la complémentarité, lorsque les autorités provinciales facilitent l'exécution des décisions de censure, et la concurrence lorsque les inspecteurs de presse se trouvent dépossédés de leur charge par la multiplication des arrêtés des gouverneurs. Entre 1905 et 1910, pour 224 fermetures de publications périodiques à la suite d'une condamnation judiciaire instruite par la censure, on décompte 1046 journaux et revues suspendus par décision administrative ${ }^{44}$.

L'emprise croissante des administrations provinciales sur le contrôle de la presse est accentuée par l'inertie des structures de la censure. On recense 78 inspecteurs de presse en 1911 contre 77 censeurs à la veille de la réforme répartis dans 8 comités rassemblant de 2 à 10 censeurs assistés de personnel de chancellerie ${ }^{45}$, et

Obščaja vnerajonnaja perepiska o presse, 1. 131, circulaire chiffrée du Département de la police $\mathrm{n}^{\circ} 170$ du $1^{\text {er juin } 1907 .}$

40. Walkin, «Government Control... », p. 208-209; Ferenczi, «Freedom of the Press... », p. 197 ; Daly, « Pressa i gosudarstvo... », p. 30.

41. GARF, f. 102, Département de la police, op. 116, 4oe deloproizvodstvo (1907), d. 923 Obščaja vnerajonnaja perepiska o presse, 1. 142, rapport du gouverneur de de Nijni-Novgorod du 27 juin 1907 qui affirme recourir à cette procédure depuis la réforme de la censure.

42. RGIA, f. 776, GUDP, op. 16 , 2e département (1906), d. 6, correspondance, 1. 56-57, courrier du 4 décembre 1906 au département de la police rendant un avis sur les mesures à prendre sous forme d'arrêtés contre la presse périodique.

43. GARF, f. 102, Département de la police, op. 116, 4oe deloproizvodstvo (1907), d. 923, Obščaja vnerajonnaja perepiska o presse, 1. 88, circulaire du GUDP n ${ }^{\circ} 1805$ du 8 février 1907.

44. Données du journaliste de Sovremennyj mir A.B. Ventin citées par V.A. Rozenberg, « Neskol’ko cifrovyh itogov » [Éléments de bilan statistique], in Svoboda pečati pri obnovlennom stroe, p. 238.

45. Par ordre décroissant d'importance : Saint-Pétersbourg, Varsovie, Moscou, Vilno, Tiflis, Kazan, Kiev, Odessa. 
11 postes individuels dans des villes de seconde importance ${ }^{46}$. On observe au cours de cette période une infime progression du nombre total d'agents locaux grâce à la création de postes de chancellerie destinés à faire face au poids de la correspondance avec les organes judiciaires développée par les nouvelles procédures de poursuite. Ce souci préside à la transformation entre 1906 et 1909 des postes de censeur de Kiev, Kazan et Odessa en comités aux affaires de presse rassemblant désormais deux à trois fonctionnaires assistés d'un secrétariat ${ }^{47}$. Mais il faut souligner que ces créations sont obtenues par un redéploiement des cadres, notamment la suppression d'emplois de censeurs des publications importées de l'étranger. Le nombre total de fonctionnaires de l'Administration générale des affaires de presse se contracte en effet entre 1905 et 1911, passant de 113 à 111 emplois ${ }^{48}$. Alors que la réforme accélère de façon notable le développement de l'activité éditoriale, la censure conserve donc pour l'essentiel les structures de 1865, lorsqu'elle fut transférée de la compétence du ministère de l'Instruction publique à celui de l'Intérieur. En dehors des vingt villes où siègent des inspecteurs de presse, le contrôle effectif des publications est à la charge de l'administration des gouverneurs et de la police. Cette inertie apparaît d'autant plus spectaculaire si on la rapporte au développement de la presse : selon la comptabilité officielle, le nombre de publications périodiques passe de 1074 titres au 26 novembre 1905 à plus de 2000 en 1910, près de 3000 en $1913^{49}$, le nombre d'ouvriers typographes augmente à Moscou de $45 \%$ entre 1906 et 1913, à Saint-Pétersbourg de $29 \%$ entre 1908 et $1913^{50}$. L'explosion du volume des imprimés et la multiplication des lieux de publication provoquée par l'abolition de la censure préventive et des autorisations préalables avaient cependant été pressenties par les autorités. Le représentant du ministère de l'Intérieur aux travaux de la commission Kobeko assurait ainsi que la réforme serait accompagnée de la création de charges de censeurs dans un total de quarante-neuf villes ${ }^{51}$. Le statu quo ne

46. Riga, Jur'ev (Dorpat), Revel, Lodz', Har'kov, Ekaterinoslav, Rostov-na-Donu, NižnijNovgorod, Saratov, Baku, Tomsk, Vladivostok. Ustav o cenzure i pečati. Izdanie neoficial'noe [Statut de la censure et de la presse. Édition non officielle], SPb : sans éditeur connu, [19111912], p. 255-258.

47. Patruševa, «Istorija cenzurnyh... », p. 15 ; V. V. Antonov et alii, « Cenzory Malorossii, Novorossii i Slobodskoj Ukrainy v XIX-načale Xx veka» [Les censeurs de Petite Russie, Nouvelle Russie et d'Ukraine septentrionale à la fin du XIX ${ }^{\mathrm{e}}$-début du XX ${ }^{\mathrm{e}} \mathrm{s}$.], Knižnoe delo $v$ Rossii v XIX -načale XX veka, 13, 2006, p. 191-192.

48. Nous avons systématisé les données fournies par N.G. Patruševa (« Istorija cenzurnyh... », p. 33-41) pour le début de l'année 1905 de façon à les comparer aux listes du personnel fournies dans Ustav o cenzure ...[1911-1912], p. 255-258. Pour permettre la comparaison, les emplois de copistes (pis'movoditeli) n'ont pas été pris en compte.

49. S.Ja. Mahonina, Russkaja dorevoljucionnaja pečat' (1905-1914) [La presse russe d'avant la révolution,1905-1914], M. : MGU, 1991, p. 11-12. Ces données ne tiennent pas compte de la Finlande.

50. Victoria Bonnell, Roots of Rebellion : Workers' Politics and Organziations in St. Petersburg and Moscow, 1900-1914, p. 362-363. Il s'agit des statistiques de l'Inspection des fabriques, qui ne tiennent pas compte des imprimeries de l'État.

51. Patruševa, «Istorija cenzurnyh... », p. 15 ; Gilbert Doctorow, «Reforma carskoj cenzury »,p. 193. 
s'explique pas seulement par des questions financières, puisque Witte obtient de l'empereur un crédit supplémentaire de 150000 roubles en mars 1906 pour le financement de la censure ${ }^{52}$ portant son budget annuel global à un peu plus de 480000 roubles $^{53}$. Les débats du Conseil d'État et du cabinet des ministres laissent croire à un arbitrage politique entre partisans de la réforme appuyés par Kobeko et conservateurs. Les premiers se rangent au principe de la simultanéité du dépôt légal des publications auprès de la censure avec leur diffusion commerciale au prix d'un rejet du monopole des Comités aux affaires de presse sur le contrôle des publications non périodiques ${ }^{54}$. Ils refusent que leurs compétences s'étendent hors de leur ville d'implantation où le contrôle des imprimés reste ainsi délégué à la police locale sous l'autorité des gouverneurs. L'inertie des cadres de la censure est ainsi un moyen de limiter l'ampleur de la réforme au profit d'un contrôle policier.

Les organes de contrôle de la presse s'efforcent toutefois d'asseoir leur légitimité en faisant évoluer leur mission. Ces efforts incitent à relativiser la crise que laissent transparaître les difficultés du contrôle de la presse.

\section{La censure à la recherche d'une légitimité}

L'Administration des affaires de presse développe deux stratégies pour répondre à la double contestation que constituent les critiques de la presse et la concurrence du pouvoir policier.

La première consiste à défendre une action de l'État fondée sur le droit. Cette posture s'observe notamment dans les villes de province où la censure est exercée par les autorités provinciales et les fonctionnaires du Département de la police. Les poursuites y sont trop souvent motivées par des accusations vagues alors que les circulaires ministérielles exigent des références précises aux textes pénaux pour définir les textes incriminés ${ }^{55}$. Ces instructions révèlent le manque de familiarité avec les normes juridiques de ces agents provinciaux et défendent une application stricte de la réglementation. À l'inverse, les inspecteurs des comités des grandes villes, qui sont directement rattachés à l'Administration centrale et jouissent d'une réelle autonomie à l'égard des autorités de police locale, semblent être marqués par une formation juridique poussée. Ces derniers ne sont plus des savants, des universitaires ou des hommes de lettres, comme cela était fréquent dans la première moitié du XIX $\mathrm{X}^{\mathrm{e}}$ siècle lorsque la censure était rattachée au ministère de l'Instruction publique. Ils sont à $40 \%$ issus des facultés de droit et ont souvent entamé leur

52. M. V. Maškova, M. V. Sokurova, « Iz istorii vozniknovenija Knižnoj letopisi » [Éléments sur la création des Annales du livre], Sovetskaja bibliografija,47, 1957, p. 15.

53. Patruševa, « Istorija cenzurnyj učreždenij... », p. 16.

54. OR RNB, f. 781, d. 296, 1. 27-28, compte rendu des débats du Conseil d'État du 4 avril 1906.

55. GARF, f. 102, Département de la police, op. 120, 4oe deloproizvodstvo (1911), d. 100¹, 1. 10 et 148, circulaires du GUDP des 1 ${ }^{\text {er }}$ février et 4 novembre 1911. 
carrière dans les services du ministère des Finances et de la Justice pour les censeurs de la capitale, dans les chancelleries provinciales dans les autres villes. Aleksej A. Sidorov, qui prend la tête du Comité aux affaires de presse de Moscou en 1909 est un bon exemple de ce profil d'administrateur. Noble, diplômé de droit à l'université de Varsovie, il occupe différents postes auprès du gouverneur de Varsovie et entre dans la censure à l'âge de 29 ans en 1893. Titularisé au bout de six ans, il est chargé de présider le nouveau comité de Kiev en 1906 avant d'être finalement promu à Moscou et nommé cadre de l'Administration centrale au début de la guerre $^{56}$. Un autre trait marquant est l'émergence de fonctionnaires qui n'ont pas accompli d'études universitaires, mais ont commencé aux environs de leurs vingt ans à occuper des postes de chancellerie peu élevés avant de gravir progressivement les échelons de la carrière jusqu'à des postes de censeur parfois prestigieux. À Odessa, Dmitrij I. Bezpal'ko, fils de paysan, collabore avec la censure dès l'âge de 15 ans en 1884, exerçant sous contrat diverses menues tâches avant de se voir confier, au bout de vingt-cinq ans, la fonction de secrétaire du comité et de participer à partir de 1913 aux réunions avec le rang de censeur ${ }^{57}$. La censure est donc marquée au début du siècle par le choix d'administrateurs expérimentés qui effectuent l'essentiel de leur carrière au sein de l'Administration des affaires de presse. Le métier de censeur n'est plus, comme au milieu du siècle précédent, une étape temporaire avant des postes plus prestigieux au sein du ministère de l'Intérieur, même s'il débouche parfois sur des postes de cadre au sein de l'administration centrale dont les responsables sont le plus souvent recrutés parmi les inspecteurs de presse $^{58}$. Cette situation a certainement contribué à l'émergence d'une identité professionnelle jalouse de l'indépendance des comités aux affaires de presse à l'égard des autorités locales et du département de la police.

Les réformes de statut de 1850 , le tournant libéral de 1855 et le transfert de la censure du ministère de l'Instruction publique au ministère de l'Intérieur en 18631865 avaient suscité des crises accompagnées de départs volontaires ou d'évictions ${ }^{59}$. À l'inverse, le changement d'orientation politique en matière de presse ne provoque pas en 1905 de mouvements notables de personnel. À Moscou et SaintPétersbourg en 1906 et 1907, on dénombre seulement trois recrutements qui correspondent à des vacances provoquées par des décès ${ }^{60}$. À son arrivée à Moscou en 1909, A.A. Sidorov, qui est nommé avec la mission de défendre l'autonomie de la censure à l'égard du gouverneur, s'attendait à rencontrer de nombreuses difficultés

56. N.A. Gričenko, N.G. Patruševa, « Cenzory Moskvy : 1804-1917 » [Les censeurs de Moscou : 1804-1917], Novoe Literaturnoe Obozrenie, 2000, 44, p. 418.

57. Antonov et alii, « Cenzory Malorossii... », p. 224.

58. Irving P. Foote, «The St. Petersburg Censorship Committee, 1828-1905», Oxford Slavonic Papers, 24, 1991, p. 78-80.

59. Foote, « The St. Petersburg Censorship Committee...», p. 73-77, 81.

60. Les mouvements du personnel ont été reconstitués à partir des notices biographiques publiées par Patruševa et Gričenko : «Cenzory Moskvy... », p. 409-433 ; «Cenzory Peterburga (1801-1917)» [Les censeurs de Saint-Pétersbourg (1804-1917)], Novoe Literaturnoe Obozrenie, 2004, 69, p. 364-394. 
parmi ses collaborateurs. Il ne relève toutefois qu'une seule tête brûlée, le censeur Sergej I. Sokolov, âgé de 57 ans dont il note néanmoins que la culture de soumission à la hiérarchie l'emporte sur l'hostilité à la réforme ${ }^{61}$.

Face aux organes de police agissant dans le cadre des lois d'exception, l'Administration des affaires de presse agit comme la vitrine d'une administration soucieuse du droit et de la légalité. Cette démarche est une conséquence directe de la censure répressive. Le travail de contrôle ne se déroule plus dans le secret des bureaux sur des manuscrits encore confidentiels mais s'exerce sur des publications qui ont par ailleurs l'occasion de circuler dans la société. N.V. Šahovskoj avait attiré l'attention de la commission Kobeko sur cette évolution prévisible : « Les nouvelles lois sur la presse feront que, de plus en plus, les organes de contrôle répondront de leur activité non pas devant le gouvernement mais devant l'opinion [obščestvo] et se trouveront plus exposés qu'aujourd'hui ${ }^{62}$. La censure ne se présente pas comme un organe répressif destiné à empêcher le développement d'une sphère publique mais comme un agent de régulation et de promotion d'une liberté d'expression qui a besoin de l'encadrement de l'État pour se développer. La libéralisation de la législation est perçue comme un danger, non pas du seul point de vue du pouvoir politique, mais parce qu'elle rend l'opinion publique prisonnière des spéculations commerciales, de la démagogie politique et des surenchères révolutionnaires. Les fonctionnaires sont en effet surpris de la rapidité avec laquelle la presse profite du nouveau contexte règlementaire pour se diversifier : l'essor spectaculaire de la presse satirique est ainsi qualifié de «cauchemar» par le censeur pétersbourgeois Umanec ${ }^{63}$. Les responsables de la censure regrettent dès avril 1905, dans une note confidentielle, que les développements récents de la presse aient révélé que «l'opinion publique n'est qu'un magma confus d'idées ${ }^{64}$. L'Administration générale des affaires de presse s'attache ainsi dès 1905 à intervenir dans l'organisation de la presse au nom d'un principe d'objectivité. A.V. Lihomanov a bien étudié les efforts, sous S.Ju. Witte et P.A. Stolypin, pour créer un organe de presse officiel chargé d'exposer le point de vue du gouvernement tout en s'ouvrant à des prises de position critiques. Le sort de ce journal, Russkoe gosudarstvo, lancé en février 1906 est cependant un échec en raison de l'indifférence du public et la censure privilégie une politique de subsides secrètes à différents périodiques, notamment Rossija qui devient l'organe officieux du pouvoir ${ }^{65}$. La transformation de la revue Sel'skij

61. A.A. Sidorov, «Iz zapisok moskovskogo cenzora (1909-1917) [ [Notes d'un censeur moscovite, 1909-1917], Golos minuvšego, VI, 1-3, janvier-mars 1918, p. 98.

62. Cité par N.V. Maškova, Istorija russkoj bibliografii načala XX veka (do oktjabrja 1917 g.) [Histoire de la bibliographie russe au début du XXe siècle, jusqu'en octobre 1917], M. : Kniga, 1969, p. 46.

63. Umanec, « Iz prošlogo našej cenzury », p. 201.

64. RGIA, f. 776, GUDP, op. 22 (1905), IVe département, d. 70, Projets et documents relatifs au statut de la presse, 1.1 verso, rapport du 26 avril 1905.

65. A.V. Lihomanov, Bor'ba samoderžavija za obščestvennoe mnenie, 1905-1907 [L'autocratie à la conquête de l'opinion publique], SPb. : RNB, 1997, 133 p. 
vestnik, destinée aux campagnes et financée par le ministère de l'Intérieur depuis 1881, est plus aboutie. Le titre change de maquette et de format pour perdre son caractère officiel au profit d'articles plus diversifiés, d'éditoriaux et augmente sa périodicité au prix, cependant, d'une contraction de la diffusion qui stagne autour de 100000 exemplaires. À partir de 1910, le titre se dote d'une structure éditoriale appuyée sur le réseau de distribution de la grande firme moscovite I.D. Sytin, spécialisée dans les publications populaires à grand tirage. À l'occasion de l'anniversiaire de la campagne de 1812 et du tricentenaire de la dynastie des Romanov, plusieurs centaines de milliers de brochures et de portraits sont ainsi diffusés dans les campagnes $^{66}$. L'Administration des affaires de presse est le principal artisan de cette politique. La censure cherche ainsi à façonner une opinion publique conforme aux attentes du gouvernement par des méthodes incitatives et non seulement dans une logique répressive.

La censure développe également un discours minorant sa dimension politique au profit d'une image d'observateur impartial du monde de la presse dont on trouve les échos dans les initiatives prises par l'Administration générale des affaires de presse après 1906.

Il s'agit, en premier lieu, de la constitution d'une bibliographie scientifique nationale, qui donne naissance, à partir de juillet 1907, à l'hebdomadaire Knižnaja letopis' (Annales du livre) s'efforçant de recenser et de décrire l'ensemble des publications de l'Empire ${ }^{67}$. À la différence des listes d'ouvrages recommandés par le pouvoir, autorisés dans les bibliothèques populaires ou des listes d'ouvrages interdits dans les cabinets de lecture et mis à l'index que la censure a multipliées jusqu'en 1905, cette bibliographie veut être un outil de recensement et de célébration de la richesse et de la diversité de la production imprimée en Russie obéissant à un but «pratique et scientifique ${ }^{68}$. Cette démarche est prolongée par l'organisation à partir de 1908 d'Expositions de la presse russe qui se tiennent dans les locaux du ministère de l'Intérieur. Il s'agit d'une manifestation à caractère patriotique, destinée à célébrer la richesse d'un secteur économique et de l'activité intellectuelle du pays. Le discours de ces expositions met en avant une volonté d'objectivité scientifique. Le but n'est pas de présenter aux visiteurs une sélection des ouvrages et publications valorisés par le pouvoir mais de donner une image exhaustive de l'activité éditoriale du pays. Le catalogue précise ainsi qu'il s'agit de « donner une idée réelle et impartiale de la presse russe, sans en excepter les organes opposants, même subversifs ou immoraux, même ceux qui ont pu donner lieu à des poursuites

66. James H. Krukones, To the People : the Russian Government and the Newspaper Sel'skij vestnik (Village Herald) 1881-1917, New York: Garland Publishing Inc., 1987, p. 191-213; Richard Wortman, Scenarios of Power : Myths and Ceremony in the Russian Monarchy, vol. 2, Princeton : Princeton U.P., 2000, p. 488-489.

67. B.A. Semenovker, Gosudarstvennaja bibliografija Rossii XVIII- XX VV. Peterburgskij period [La bibliographie nationale russe, $\mathrm{XVIII}^{\mathrm{e}}-\mathrm{XX}$ e s. : la période pétersbourgeoise], M. : Paškov dom, 2002, vol. 2, p. 6-42.

68. OR RNB, f. 787, fonds A.V. Toropov, op. 1, d. 9, 1. 4, circulaire de 1'Administration générale des imprimés du 8 juillet 1907 nº 7147. 
légales ou à des ordres d'interdiction ${ }^{69}$. Dans les salles d'exposition, des tables regroupent aussi bien les revues d'art et la grande presse quotidienne que les revues satiriques d'opposition et les publications grivoises.

Le travail d'enregistrement bibliographique et d'exposition est rendu possible par les fonctions d'enregistrement de la censure. Depuis avril 1906, l'Administration des affaires de presse est chargée de centraliser le dépôt légal des nouvelles publications dont les exemplaires sont destinés aussi bien aux bibliothèques patrimoniales qu'aux procédures de contrôle ${ }^{70}$. En menant à bien l'enregistrement des imprimés, la censure sort de la stricte logique policière et cherche à donner à son travail la valeur d'un outil scientifique au service des professionnels du livre et des savants. Ce contrôle du dépôt légal lui permet ainsi de défendre une compétence exclusive sur la presse alors que son autorité a été compromise tant par les opérations de boycott que par le rôle important dévolu à la police dans le contrôle effectif des imprimés. Ainsi, le chef de l'Administration générale des affaires de presse, Aleksej V. Bel'gard, n'hésite pas à chercher le soutien de la section bibliographique de l'Académie des Sciences en avril 1906 pour défendre le monopole de la censure sur l'organisation du dépôt légal ${ }^{71}$. L'organisateur du travail de recensement des nouvelles publications, A.D. Toropov, est une figure reconnue des milieux de la bibliographie scientifique. Il se voit confier la rédaction de la Knižnaja Letopis`au printemps 1907, alors qu'il a été arrêté au mois de décembre pour avoir publié, comme rédacteur du journal Kuban', des appels à la grève et participé au boycott de la censure ${ }^{72}$. Ce parcours montre le caractère relatif de la dichotomie opposant les inspecteurs de presse au monde éditorial.

L'attitude des censeurs envers les ouvrages poursuivis peut également sembler paradoxale. Les publications condamnées ne font jamais l'objet d'une destruction totale, un petit nombre d'exemplaires est destiné aux archives du service, à celles de la police, mais également aux bibliothèques patrimoniales qui ne mettent toutefois pas ces ouvrages à la disposition du public. À Odessa, les autorités locales de la censure remettent ainsi en 1910 à la bibliothèque de la ville leur collection de brochures illégales de la période 1905-1907 afin qu'elle les conserve « pour les historiens futurs » ${ }^{73}$. Après 1905, la logique que les censeurs mettent en œuvre n'est donc pas strictement policière, elle se constitue également selon un principe patrimonial de préservation.

69. Vystavka proizvedenij pečati za 1908 g., ustroennaja Glavnym Upravleniem po delam pečati v Sankt-Peterburge s 9 maja po 9 ijunija $1909 \mathrm{~g}$. [Exposition des ouvrages de presse de l'année 1908 organisée par l'Administration générale des affaires de presse à Saint-Pétersbourg du 9 mai au 9 juin 1909], SPb. : tipografija MVD, 1909, p. 20.

70. CIAM (Central’nyj istoričeskij arhiv g. Moskvy - Archives historiques de la ville de Moscou), f. 31, Comité des affaires de presse de Moscou, op. 3, circulaires de l'Administration centrale, d. 484, 1. 3, circulaire $n^{\circ} 3058$ du 6 avril 1906.

71. Maškova, Sokurova, « Iz istorii ... », p. 16.

72. Maškova, Istorija russkoj bibliografii..., p. 48.

73. Otčet odesskoj gorodskoj imeni Imperatora Nikolaja II Publičnoj biblioteki za $1910 \mathrm{~g}$. [Compte rendu d'activité de la Bibliothèque municipale publique Nicolas II d'Odessa pour l'année 1910], Odessa : Slavjanskaja tipografija, 1911,p. 9. 


\section{Conclusion}

Les événements et la législation de 1905 sont vécus par les censeurs comme une crise car ils se voient dépossédés des moyens d'accomplir la mission de contrôle de la presse qui leur est confiée. Mis en concurrence avec les pouvoirs de police, ils doivent également répondre aux interpellations de l'opinion et se conformer à l'effort affiché de transparence et de libéralisation de régime. L'existence de la censure est ainsi justifiée par les pressions commerciales et les surenchères politiques qui pèsent sur la presse, les censeurs conçoivent alors leur rôle comme celui de régulateurs et d'organisateurs d'une opinion éclairée. La constitution d'un outil bibliographique devient ainsi un instrument de légitimation de la censure illustrant le rôle de l'État comme agent du débat public. Cela lui permet également de répondre aux critiques sur les abus de la législation en vigueur en opposant la vigueur et la diversité de la presse russe.

Ces entreprises ne suffisent cependant pas à contrebalancer la marginalisation de la censure. L'historien de la presse V. Rozenberg critique l'entreprise bibliographique qui masque selon lui l'ampleur de la répression sous la célébration de la croissance du nombre de publications ${ }^{74}$. Par ailleurs, en insistant sur leur rôle de promotion de la presse, les inspecteurs de presse prêtent le flanc aux critiques des éléments conservateurs qui privilégient une approche policière et critiquent la rigidité des règles instaurées au printemps 1906. Ces tensions contradictoires expliquent également que la réforme de la loi sur la presse reste jusqu'à la chute du régime un véritable serpent de mer de la vie politique russe.

Université Paris-I

Centre de recherche sur l'histoire des Slaves

guichard.b@gmail.com

74. Rozenberg, « Neskol’ko cifrovyh... », p. 225-244. 